"This is an original manuscript of the article published by: H. Malekmohammadi, S. Laureti, M. Ricci, Q. Yi, J. Zhu, G. Y. Tian, in '2018 IEEE Far East NDT New Technology \& Application Forum (FENDT)', 6-8 July 2018.

Available online at: https://ieeexplore.ieee.org/document/8681968 DOI: 10.1109/FENDT.2018.8681968

“C 2018 IEEE. Personal use of this material is permitted. Permission from IEEE must be obtained for all other uses, in any current or future media, including reprinting/republishing this material for advertising or promotional purposes, creating new collective works, for resale or redistribution to servers or lists, or reuse of any copyrighted component of this work in other works." 


\title{
An Experimental Comparison of LED and Eddy Current Pulse-Compression Thermography on an Impact Damage CFRP Benchmark Sample
}

\author{
Hamed Malekmohammadi ${ }^{1}$, Stefano Laureti ${ }^{*}$, Marco Ricci ${ }^{2}$, Qiuji $\mathbf{Y i}^{3}$, Junzhen Zhu $^{3}$, Gui Yun Tian \\ ${ }^{1}$ Departmentof Engineering, University of Perugia, Polo Scientifico Didattico di Terni, 05100, Italy \\ ${ }^{2}$ Department of Informatics, Modeling, Electronics and System Engineering, University of Calabria, Rende, 87036, Italy \\ ${ }^{3}$ Department of Electrical and Electronic Engineering, Newcastle University, Newcastle, UK \\ Corresponding author: stefano.laureti@unipg.it
}

\begin{abstract}
A comparison of the application of Pulse-Compression to eddy-current and LED thermography is presented. Results achieved on a dedicated sample with an impact damage are reported and discussed. The same pulse compression procedure based on the use of Barker codes has been applied to both techniques and it proved to be work properly in both cases. Two different features have been extracted and imaged from the retrieved time-responses after pulse-compression.
\end{abstract}

Keywords: Non-destructive Test; Pulse-Compression; IR Thermography; Eddy Current Pulsed Thermography; Pulse compression Thermography; Impact damage; CFRP; Coded signals

\section{Introduction}

Active Thermography (AT) is a non-destructive testing (NDT) technique extensively exploited in research and industrial applications not only for detect eventual defects in the Sample Under Test (SUT) but also for material characterization [1-3], cultural heritage diagnostic [4-6], on-line monitoring of goods in different production plants [7-9], etc.

AT always relies on the application of an external heating stimulus over the SUT to reach the desired thermal contrast [10]. In most of the applications, the heating stimulus is realized with some light source such as flashlamps, quartz lamps linear systems halogen lamps, laser heating and LED chips [11-18]. In all these cases, the heat is firstly transferred to the illuminated sample surface and then it diffuses inside the sample [19]. In addition to lightbased AT, alternative methods of thermal excitation have been proposed and are currently applied, which are based on phenomena used in other NDT techniques: Ultrasound Vibro-Thermography [20], Eddy-current Pulsed Thermography (ECPT) [21], Microwave Thermography [22]. With respect to the light-based techniques, in these cases the heat can be provided directly to the whole sample volume (bulk heating) and, in some cases, the heat can be selectively generated only in presence of an inhomogeneity or of a defect [23-24].

Despite the employed heating source, the classification of the existing AT schemes is strictly related to the timefrequency characteristic of the heating modulation signal. In Pulsed Thermography (PT), a short time duration heating stimulus that well-approximates the Dirac's delta $\delta(t)$ excites the SUT. Features of interest can be extracted by analysing both the heating and the cooling trend of the recorded impulse response $h(t)$ pixelwise. It is worth to note that the maximum achievable Signal-to-Noise Ratio (SNR) in PT is set by the excitation source power. Improved SNR values can be obtained by modulating the heating stimulus with a sinusoid at given frequency, i.e. Lock-in Thermography (LT) at the cost of less information with respect to the one obtainable by using PT [25-26]. In the recent years, efforts have been made to merge effectiveness of PT and the SNR values achievable via LT, leading to Pulsed Phase Thermography and MultiFrequency Lock-in Thermography. To this aim, the use of a coded signal to modulate the heating stimulus together with Pulse Compression $(\mathrm{PuC})$ to retrieve the sample impulse response has been also investigated [27-32]. In Pulse Compression Thermography (PuCT), the heating emission is commonly modulated either by a phasemodulated signal, e.g. Barker code, or by a frequencymodulated "Chirp" signal. These coded signals have a unique characteristic: their duration $T$ and bandwidth $B$ are uncorrelated. Thus, the signal frequency content can be designed to suit the investigation of a given sample, while its duration can be enhanced almost arbitrarily to reach the desired SNR [33]. Although PuCT is now a quite established technique, very few attempts are reported in literature to extend this approach to Eddy-Current Thermography [34]. To further investigate this possibility, in this paper experiments were carried out both with Eddy-Current Pulse Compression Thermography (ECPuCT) and with LED-stimulated PuCT on an impact damaged Carbon Fibre Reinforced Plastic (CFRP) benchmark sample described in detail in Section 3.3. The results are then compared to evaluate the effectiveness and the feasibility of Eddy-Current Pulse Compression Thermography (ECPuCT) with respect to LED-stimulated PuCT. Evalua- 
tion of the impact damage on the composite materials using ECPT have been reported in literature [35-37], and the use of various feature extraction techniques for automatic detection and classification has been also reported [38]. Here the main goal is to apply to ECPT the PulseCompression procedure described in [18] and analyse the performances achieved compared by those provided by LED-PuCT. To do this, Barker code [39] signal was employed to drive the two different heating systems: an induction coil (ECPT) and LED chips. Although the physics underlying the two exploited approaches is different, i.e. heat dissipation by Joule's effect and volumetric heating for the Eddy Current case with respect to the ideallyconsidered 1D diffusion in the LED case, it is shown that $\mathrm{PuC}$ is a robust strategy that could be exploited in both cases. In addition, the suitability of the non-linear fitting approach for subtracting the step heating contribution proposed for LED PuCT in [18] was successfully verified for the proposed ECPuCT scheme.

\section{Introduction to Pulse Compression}

The PuC algorithm has been exploited on two different setups, which are described in detail in Section 3. To gain insight on how the PuC can be successfully implemented for the two setups, a brief introduction about the mathematical theory of the PuC technique is given here. The reader is suggested to refer for example to [33] for further details. PuC is a powerful measurement technique widely used to estimate the impulse response $h(t)$ of a LinearTime Invariant (LTI) system in poor SNR conditions. Given a pair of coded signals, the excitation $s(t)$ that modulates the heating source, and the so-called matched filter $\psi(t)$, such that their convolution (denoted by *) approximates the Dirac's Delta function $\delta(t)$, $s(t) * \psi(t)=\delta(t) \cong \delta(t)$, an estimate $\tilde{h}(t)$ of $h(t)$ can be obtained by exciting the LTI system with $s(t)$ and then by convolving the system output $y(t)$ with $\psi(t)$. This is demonstrated in (1) and in Fig. 6 for a single pixel of the acquired thermogram. The presence of an Additive White Gaussian Noise (AWGN) as the $e(t)$ term, uncorrelated to $\psi(t)$, is considered. Note that the SUT is assumed to be an LTI system.

$$
\begin{aligned}
& \tilde{h}(t)=y(t) * \psi(t)=h(t) * \underbrace{s(t) * \psi(t)}_{=\tilde{E}(t)}+\epsilon(t) * \psi(t) \\
& h(t) * \tilde{\delta}(t)+\tilde{e}(t) \approx h(t)+\tilde{e}(t)
\end{aligned}
$$

The main pros of $\mathrm{PuC}$ is directly related to the peculiar characteristics of the coded signal $s(t)$ used, which has uncorrelated $T$ and $B$. It can be demonstrated that the SNR gain achieved by using $\mathrm{PuC}$ with respect to a pulsed excitation of the same peak power is $\sim T \times B$. Consequently, the higher is the $T \times B$ product, the closer is the approximation of the Dirac's Delta function: the amplitude of the sidelobes of $\delta(t)$, which affect $\tilde{h}(t)$, decreases as $T \times B$ increases. In this work, the employed coded signal for mod-

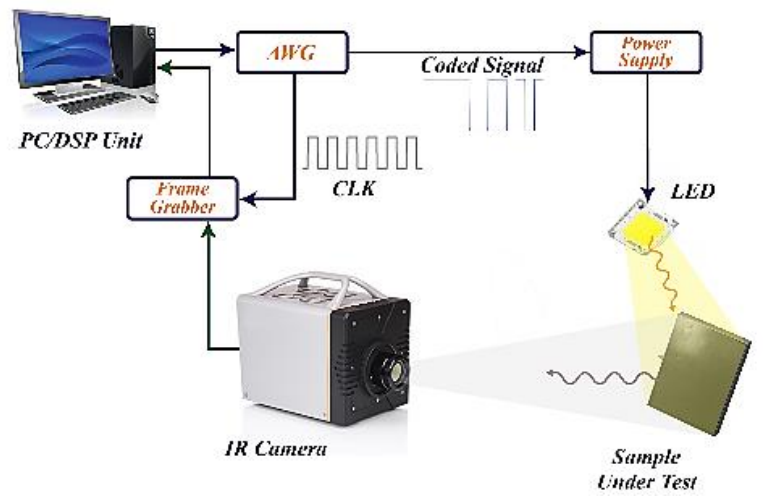

Fig. 1. Block diagram of the LED experimental setup.

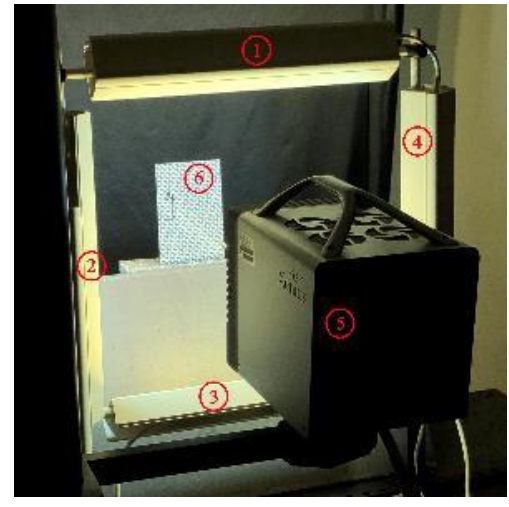

Fig. 2. LED experimental setup: (1) to (4) LED chips, (5) IR camera, (6) SUT.

ulating the heat sources was a Barker Code (BC) [39-40] with a bit length of 13 , that in turn assures sidelobe level suppression of $22.3 \mathrm{~dB}$. The BC signal used is plotted versus time in Fig. 5(a), in which each original bit lasts one second at the chosen frame rates used for the ECPuCT and the PuCT setups, as described in Sections 3.1 and 3.2. Note, that also the SUT cooling trend was acquired for additional thirty seconds after the ending of the BC. Although the physics underlying the heat diffusion on the two setups is different, the chosen padding duration onsets thermal waves with increased thermal diffusion wavelength, thus assuring enough sensitivity at deeper depth within the inspected SUT [10,19, 24].

\section{Experimental Setup}

In this Section, the experimental setup and exploited signals are shown and described for both methods.

\subsection{LED PuCT}

The setup used in this experiment was the same introduced in $[6,18,41]$. A 'National Instrument PCI-6711 Arbitrary Waveform Generator (AWG) board and a National Instrument 1433 Camera Link Frame Grabber were connected to a PC, where an ad-hoc developed virtual instrument (VI) in LabVIEW managed the signal generation and acquisition. The AWG board provided both the wanted excitation signal, i.e. Barker code, and a reference 


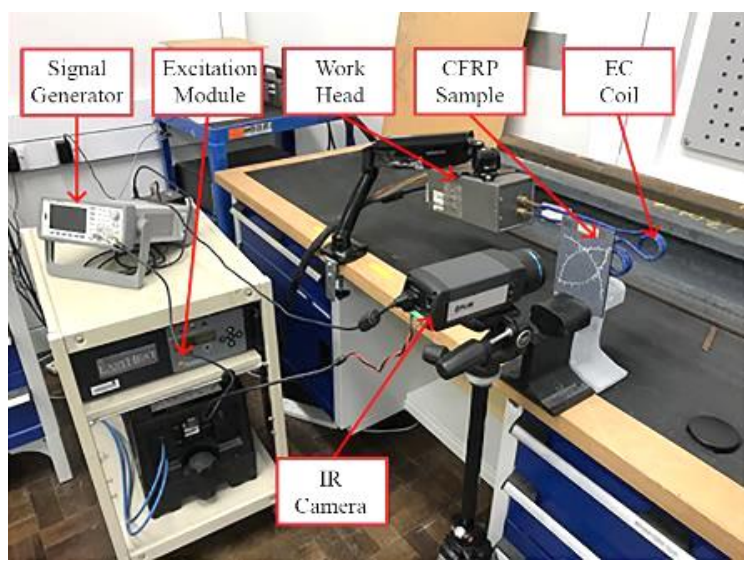

Fig. 3. ECPuCT experimental setup.

clock signal (CLK) for triggering the IR camera acquisition. The camera used was a 'Xenics Onca' equipped with an InSb cooled detector with the spectral range of 3.6 to $4.9 \mu \mathrm{m}$. Camera offers a resolution of $320 \times 256$ pixels and NETD $<17 \mathrm{mK}$. The coded signal was input into a 'TDK Lambda GEN 750W' power supply that fed eight LED chips placed at about $30 \mathrm{~cm}$ from the SUT. The LED chips are capable to provide a maximum overall power of $400 \mathrm{~W}$. The thermograms were acquired at the frame rate of $40 \mathrm{~Hz}$. A block diagram of the experimental setup used is depicted in Fig. 1, whilst Fig. 2 shows a picture of the SUT and LED setup.

\subsection{Eddy Current PuCT}

The ECPT configuration used in this experiment is shown in Fig. 3. It mainly consists of 5 units, i.e. a heating module, an induction coil, a signal generator, an infrared (IR) camera and a PC. In this work, the heating module was Easyheat 224 from Cheltenham Induction Heating with the working frequency of $260 \mathrm{kHz}$ and RMS current of $300 \mathrm{~A}$; one side of a rectangle coil was used; the signal generator was used for synchronously triggering the heating module and IR camera; the type of IR camera was the FLIR SC655 equipped with an uncooled microbolometer detector array with the resolution of 640 $\times 480$ pixels, the spectral range of $7.5-14.0 \mu \mathrm{m}$ and NETD $<30 \mathrm{mK}$. The IR camera records the surface temperature distribution of the 13 seconds excitation period as well as additional 30 seconds cooling period at 50 FPS.

\subsection{Sample Under Test}

The SUT (Fig. 4) was a quasi-isotropic CFRP plates made by Tencate (Almelo, The Netherlands), having 150 $\times 100 \times 4.2 \mathrm{~mm}^{3}$, containing 12 layers of five harness satin carbon fibers woven with balanced woven fabric. The matrix was Polyphenylene Sulfide (PPS), a thermoplastic polymer consisting of aromatic rings linked with sulfide moieties, resistant to chemical and thermal attack. The carbon fibers are T300JB type and their volume is 0.5 \pm 0.03 and the density is $1460 \mathrm{~kg} / \mathrm{m}^{3}$. The specimen was subjected to an impact of $8 \mathrm{~J}$ of energy. The impact was
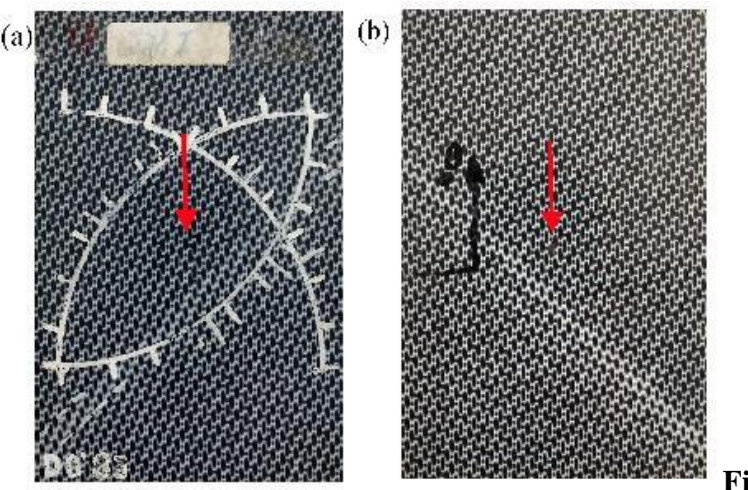

g. 4. Picture of the sample under test. (a) front side of the sample, (b) Back side of the sample. Red arrows indicate the imnact noint on hoth surfaces.

made by using FRACTOVIS PLUS 9350-CEAST instrument (Instron, Norwood, MA, USA) with a hemispherical bumper head having $20 \mathrm{~mm}$ diameter and a mass equal to $2.045 \mathrm{~kg}$. The sample exhibits electric properties that depend on the type of carbon fibers and on their volume fraction in the material. The transverse electric conductivity value was in the range [10-100] S/m, whilst the longitudinal conductivity varies between $5 \times$ $10^{3} \mathrm{~S} / \mathrm{m}$ and $5 \times 10^{4} \mathrm{~S} / \mathrm{m}$.

\section{Experimental results}

As both the heating sources are monopolar, i.e. LED chips and induction coil, a step-heating contribution must be removed from the acquired thermograms to successfully implement the PuC algorithm, as for (1). To gain insight on this, Fig. 5(a) shows the BC modulation signal, whilst the colored line plot of figures 5(b) and 5(c) depict the corresponding acquired raw signals from a single pixel on the SUT, for the PuCT and ECPuCT re-
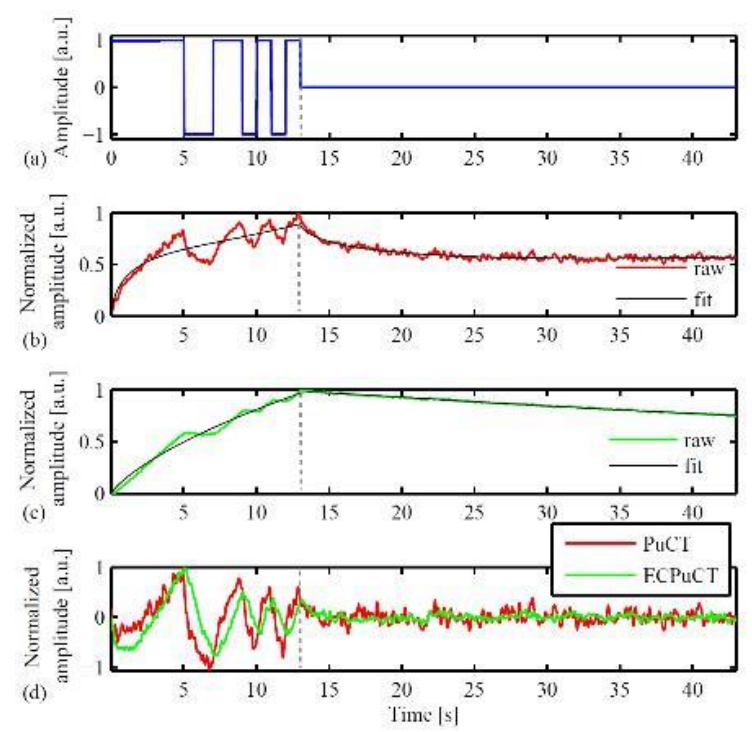

Fig. 5. An overview of the signals: (a) Barker coded signal used for heat modulation, (b) raw and fitted data for detrend of LED PuCT, (c) raw and fitted data for detrend of ECPuCT, (d) comparison of the detrended signals for LED PuCT and ECPuCT 


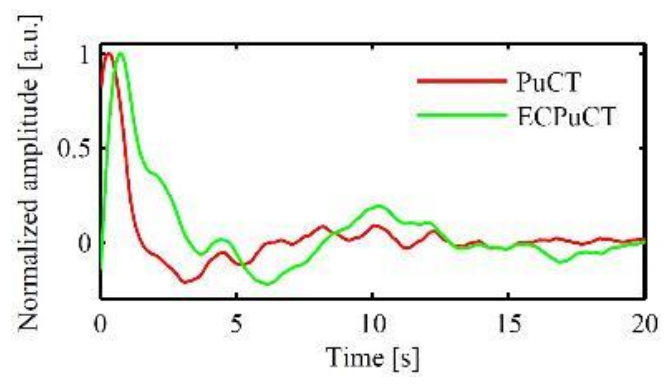

Fig. 6. Estimated impulse response of a single pixel after PuCT process with LED and ECPuCT

spectively. Recently, Silipigni et al [18] proposed an optimized strategy based on a non-linear polynomial fitting for successfully subtracting the step-heating contribution in PuCT. This strategy has been applied here also on the ECPuCT data and the resulting fitting polynomial is shown as a black line plot in Fig. 5(c). It can be appreciated from Fig. 5(d) that the proposed approach allows the step-heating contribution to be faithfully removed from the raw data, thus the PuC algorithm being effectively carried out pixelwise as for (1). Fig. 6 depicts the $\widetilde{h}(t) \mathrm{s}$ obtained from a single pixel onto the impact damaged area of the SUT for PuCT (red line plot) and ECPuCT (green line plot). It can be noted that the $h(t) \mathrm{s}$ are wellretrieved for both cases. In fact, the $\widetilde{h}(t) \mathrm{s}$ follows an exponential decay trend as time elapses (neglecting fluctuation due to sidelobes), thus in a good agreement with the one expected from a pulsed excitation.

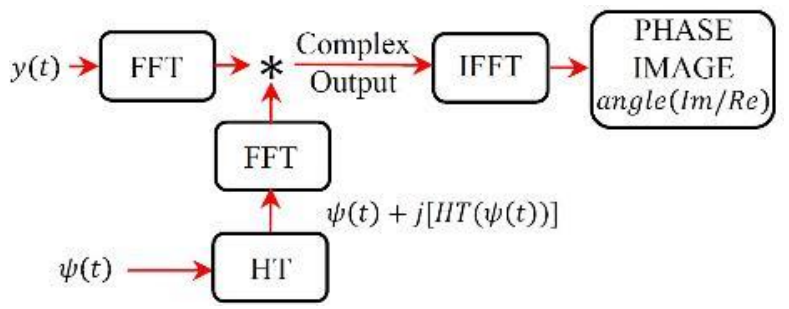

Fig. 7. PuC algorithm applied to obtain time analysis

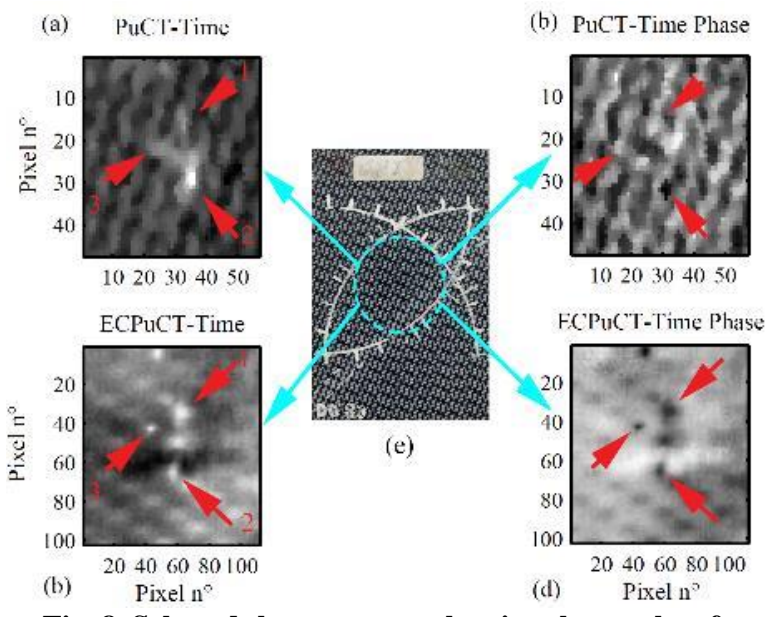

Fig. 8. Selected thermograms showing the results of PuCT and ECPuCT techniques. Red arrows show the impact damage zones detected in both methods (a) PuC time analysis, (b) PuC time-phase analysis, (c) ECPuCT time and (d) ECPuCT time-phase analysis

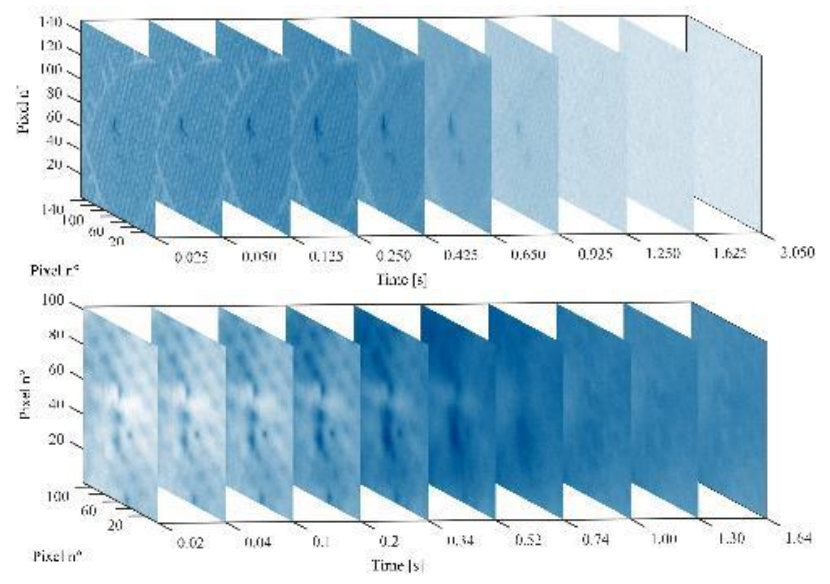

Fig. 9. (top) PuCT TIME analysis at different; (bottom) ECPuCT TIME analysis at different times (corresponding to linear inspection depths). With both methods, around $\mathrm{t}=0.5$ $s$, the TIME image depicts very well the signature of the impact defect but it does not show the surface characteristics of the CFPR. This assures a high contrast for the defect signal and an easier automatic detection

Thermograms of the sample are also shown, obtained by imaging the pixelwise amplitude of (i) the retrieved $\widetilde{h}(t)$ after performing PuC (as for (1)) and of (ii) the Time-Phase algorithm output [29-31]. The last one relies on the application of the Hilbert Transform (HT) over $\psi(t)$ before performing the PuC algorithm, as schematically reported in Fig. 7. Results obtained as for (i) will be hereinafter referred as "Time", whilst results from (ii) as "Time-Phase". Fig. 8 shows a zoom view of the obtained thermograms with "Time" and "Time-Phase" algorithms for ECPuCT and PuCT. The damaged portion is visible as a bright pixel area on the images. Please note that the depicted thermograms refers to a time instant equal to one second on the $\widetilde{h}(t)$. Finally, figure 9 reports for "Time" the images retrieved after PuC at non-equispaced time instant corresponding to linear spaced diffusion depth. For $t \sim 0.5 \mathrm{~s}$ surface characteristics disappear and only the signature of the impact damage can be seen with maximum contrast. This can be positively exploited to improve defect detection and classification

\section{Conclusions and Future Work}

Comparison of the experimental results shows that the $\mathrm{PuC}$ process can be successfully applied on the ECPT and that the proposed ECPuCT scheme is capable to detect defects. This benchmark study provides the basis for the future work on the ECPuCT along with more theoretical analysis and exploiting the feature extraction algorithms and quantitative merit figures like SNR.

\section{Acknowledgment}

This project has received funding from the European Union's Horizon 2020 research and innovation programme under the Marie Skłodowska-Curie grant agreement No 722134 - NDTonAIR. 


\section{References}

[1] C. Meola, G.M. Carlomagno, L. Giorleo, "The use of infrared thermography for materials characterization," J Mater Process Technol. 2004, 155:1132-1137.

[2] R. Steinberger, T.V. Leitão, E. Ladstätter, G. Pinter, W. Billinger, \& R.W. Lang, 2006, "Infrared thermographic techniques for nondestructive damage characterization of carbon fibre reinforced polymers during tensile fatigue testing," International Journal of Fatigue, 28(10), pp. 1340-1347.

[3] A. Mendioroz, R. Fuente-Dacal,E. Apiñaniz, \& A. Salazar, 2009. "Thermal diffusivity measurements of thin plates and filaments using lock-in thermography," Review of Scientific Instruments, 80(7), 074904.

[4] E. Grinzato, C. Bressan, S. Marinetti, P.G. Bison, \& C. Bonacina, 2002, "Monitoring of the Scrovegni Chapel by IR thermography," Giotto at infrared. Infrared Physics \& Technology, 43(3-5), pp. 165169.

[5] S. Sfarra, C. Ibarra-Castanedo, D. Paoletti, \& X.P. Maldague 2013, "Infrared vision inspection of cultural heritage objects from the city of L'Aquila, Italy and its surroundings," Materials Evaluation,71(5).

[6] S. Laureti, et al. "The use of pulse-compression thermography for detecting defects in paintings," 2018, NDT\&E (in press)

[7] L. Senni, M. Ricci, A. Palazzi, P. Burrascano, P. Pennisi \& F. Ghirelli, 2014, "On-line automatic detection of foreign bodies in biscuits by infrared thermography and image processing," Journal of Food Engineering, 128, pp. 146-156

[8] K. Li, G.Y. Tian, L. Cheng, A. Yin, W. Cao, \& S. Crichton, 2014, "State detection of bond wires in IGBT modules using eddy current pulsed thermography," IEEE Transactions on Power Electronics, 29(9), pp. 5000-5009.

[9] A.V. Weschenfelder, X.P. Maldague, L.M. Rocha, A.L. Schaefer, L. Saucier, \& L. Faucitano, 2013, "The use of infra-red thermography for pork quality prediction," Meat Science, 96, pp.120-125

[10] X.P. Maldague, "Theory and practice of infrared thermography for nondestructive testing," $2001 \mathrm{~J}$. Wiley series in microwave and optical engineering

[11] C. Ibarra-Castanedo, A. Bendada, X.P. Maldague, "Image and signal processing techniques in pulsed thermography," GESTS Int Trans Comput Sci Eng, 2005, 22.1 pp. 89-100.

[12] C. Maierhofer, P. Myrach, M. Reischel, H. Steinfurth, M. Röllig, and M. Kunert, „Characterizing damage in CFRP structures using flash thermography in reflection and transmission configurations," 2014, Composites Part B: Engineering, 57, pp. 35-46

[13] C. Ibarra-Castanedo, S. Sfarra, D. Ambrosini, D. Paoletti, A. Bendada, $\mathrm{X}: \mathrm{P}$. Maldague, "Diagnostics of panel paintings using holographic interferometry and pulsed thermography," 2010, Quant Infrared Thermogr J 2010, 7.1, pp. 85-114

[14] G.M. Carlomagno, C. Meola, "Comparison between thermographic techniques for frescoes NDT," 2002, NDT\&E Int, 35.8: pp. 559-565.

[15] A. Bendada, S. Sfarra, C. Ibarra-Castanedo, M. Akhloufi, J.P. Caumes, C. Pradere, J. Batsale, X.P. Maldague, "Subsurface imaging for panel paintings inspection: A comparative study of the ultraviolet, the visible, the Infrared and the terahertz spectra," 2015, OptoElectronics Rev 2015, 23.1: pp. 90-101.

[16] J.L. Bodnar, J.L. Nicolas, J.C. Candoré and V. Detalle, "Nondestructive testing by infrared thermography under random excitation and ARMA analysis," 2012, Int. J. Thermophysics, 33.10-11: 20112015

[17] J. Schlichting, C. Maierhofer and M. Kreutzbruck, "Crack sizing by laser excited thermography,” 2012, NDT \& E International, 45(1), pp. 133-140.

[18] G. Silipigni, P. Burrascano, D.A. Hutchins, S. Laureti, R. Petrucci, L. Senni, L. Torre, M. Ricci, "Optimization of the pulse compression technique applied to the infrared thermography nondestructive evaluation," 2017, NDT\&E Int, 87, pp. 100-110

[19] A. Salazar, 2006, "Energy propagation of thermal waves," European journal of physics, 27(6), 1349.

[20] A. Gleiter, G. Riegert, T. Zweschper, \& G. Busse, 2007, "Ultrasound lock-in thermography for advanced depth resolved defect selective imaging," Insight-Non-Destructive Testing and Condition Monitoring, 49(5), 272.

[21] J. Wilson, G.Y. Tian, I.Z. Abidin, S. Yang and D. Almond, "Pulsed eddy current thermography: system development and evaluation,"
2010, Insight-Non-Destructive Testing and Condition Monitoring, 52(2), pp. 87-90.

[22] D. Palumbo, F. Ancona, \& U. Galietti, 2015, "Quantitative damage evaluation of composite materials with microwave thermographic technique: feasibility and new data analysis," Meccanica, 50(2), pp. 443-459.

[23] J. Renshaw, J.C. Chen, S.D. Holland, \& R.B. Thompson 2011, "The sources of heat generation in vibrothermography," NDT \& E International, 44(8), pp. 736-739.

[24] A. Yin, B. Gao, G.Y. Tian, W.L. Woo, \& K. Li, 2013, "Physical interpretation and separation of eddy current pulsed thermography," Journal of Applied Physics, 113(6), 064101.

[25] D. Wu and G. Busse, "Lock-in thermography for nondestructive evaluation of materials," 1998, Revue générale de thermique, 37(8), pp.693-703.

[26] G. Riegert, T. Zweschper, \& G. Busse, 2004, „Lockin thermography with eddy current excitation," Quantitative InfraRed Thermography Journal, 1(1), pp. 21-32

[27] S. Tuli, R. Mulaveesala, "Defect detection by pulse compression in frequency modulated thermal wave imaging," 2005 Quantitative InfraRed Thermography Journal, 2.1, pp.41-54.

[28] R. Mulaveesala, S. Tuli, "Digitized frequency modulated thermal wave imaging for nondestructive testing," 2005, Materials Evaluation, 63.10.

[29] N. Tabatabaei, A. Mandelis, "Thermal-wave radar: A novel subsurface imaging modality with extended depth-resolution dynamic range,' 2009, Review of Scientific Instruments 2009, 80.3:034902.

[30] R. Mulaveesala, V.S. Ghali, "Coded excitation for infrared nondestructive testing of carbon fiber reinforced plastics,"2011, Review of Scientific Instruments, 82.5:054902.

[31] J. Gong, J. Liu, L. Qin, Y. Wang, "Investigation of carbon fiber reinforced polymer sheet with subsurface defects inspection using thermal-wave radar imaging based on the multi-transform technique," 2014, NDT \& E International 62, pp.130-136.

[32] V.S. Ghali, S.S:B. Panda and R. Mulaveesala, "Barker coded thermal wave imaging for defect detection in carbon fibre-reinforced plastics," 2011, Insight-Non-Destructive Testing and Condition Monitoring, 53(11), pp.621-624.

[33] D. Hutchins, P. Burrascano, L. Davis, S. Laureti, M. Ricci, "Coded waveforms for optimised air-coupled ultrasonic nondestructive evaluation," 2014, Ultrasonics 54, pp.1745-59.

[34] R. Yang, \& Y. He, 2015, "Pulsed inductive thermal wave radar using cross correlation matched filtering in eddy current thermography," Infrared Physics \& Technology, 71, pp. 469-474.

[35] W. Ren, J. Liu, G.Y. Tian, B. Gao, L. Chengì and H. Yang, Quantitative non-destructive evaluation method for impact damage using eddy current pulsed thermography," 2013, Composites Part B: Engineering, 54, pp. 169-179.

[36] T. Liang, W. Ren, G.Y. Tian, M. Elradi and Y. Gao, "Low energy impact damage detection in CFRP using eddy current pulsed thermography," 2016, Composite Structures, 143, pp. 352-361.

[37] Y. He, G. Tian, M. Pan, and D. Chen, "Impact evaluation in carbon fiber reinforced plastic (CFRP) laminates using eddy current pulsed thermography," 2014, Composite Structures, 109.

[38] L. Cheng, B. Gao, G.Y. Tian, W.L. Woo, \& G. Berthiau, "Impact damage detection and identification using eddy current pulsed thermography through integration of PCA and ICA," 2014, IEEE Sensors Journal, 14(5), pp. 1655-1663.

[39] R. Barker, "Group Synchonizing of Binary Digital Systems, Communication Theory," Academic Press, Inc., New York, 1953

[40] M.N. Cohen, M.R. Fox, and J.M. Baden, 1990, May. "Minimum peak sidelobe pulse compression codes". In Radar Conference, 1990., Record of the IEEE International (pp. 633-638). IEEE.

[41] S. Laureti, G. Silipigni, L. Senni, R. Tomasello, P. Burrascano, M. Ricci, "Comparative study between linear and non-linear frequencymodulated pulse-compression thermography," 2018, Applied Optics, 57.18: pp. D32-D39 